\title{
Valued Hesitation in intervals comparison
}

\author{
Meltem Öztürk ${ }^{1}$ and Alexis Tsoukiàs ${ }^{2}$ \\ 1 CRIL Université d'Artois, \\ ozturkecril.univ-artois.fr \\ 2 LAMSADE - CNRS, Université Paris Dauphine, \\ tsoukias@lamsade.dauphine.fr
}

\begin{abstract}
The paper presents a valued extension of the recently introduced concept of $P Q I$ interval order. The main idea is that, while comparing objects represented by interval of values there is a zone of hesitation between strict difference and strict similarity which could be modelled through valued relations. The paper presents suitable definitions of such valued relations fulfilling a number of interesting properties. The use of such a tool in data analysis and rough sets theory is discussed in the paper.
\end{abstract}

Keywords: interval orders, $P Q I$ interval orders, valued relations, valued similarity, uncertainty modelling.

\section{Introduction}

Comparing objects described under form of intervals dates back to the work of Luce, [1], where difference of utilities are perceived only when beyond a threshold (for a comprehensive discussion on the concepts of semi-order and interval order see [2,3]). The basic idea introduced in such works is that when we compare objects under form of intervals they can be considered as different (preferred) iff their associated intervals have an empty intersection. Otherwise they are similar (indifferent). However, such an approach does not distinguish the specific case where one interval is "more to the right" (in the sense of the reals) of the other, but they have a non empty intersection. Such a situation can be viewed as an hesitation (denoted $Q$ ) between preference (dissimilarity, denoted $P$ ) and indifference (similarity, denoted $I$ ) and merits a specific attention.

Recently Tsoukiàs and Vincke [4] gave a complete characterisation of such a structure (denoted as $P Q I$ interval order), while in [5] and [6] a polynomial algorithms for the detection of such a structure in an oriented graph are provided. In this paper we extend such results considering the situation of hesitation under a continuous valuation of preference and indifference. The idea is that the intersection of the two intervals can be more or less large thus resulting in a more or less large hesitation represented by a value in the interval $[0,1]$ for preference and indifference.

The paper is organised as follows. In the next section we introduce the basic notation and results on regular interval orders and semi-orders. In section 3 we introduce 
the concept of $P Q I$ interval order and semi-order. In section 4 we present a general frame work for the characterisation of preference structures with three binary relations ( $P, Q$ and $I$ ) and introduce a functional representation for preference (dissimilarity) and indifference (similarity) fulfilling a number of nice properties. Further research directions are included in the conclusions. The paper updates and extends results appeared originally in [7].

\section{Interval Orders}

In the following we will consider objects represented under form of intervals of values. Given a finite set $A$ of objects we associate to each element of $A$ two functions $l: A \mapsto \mathbb{R}$ and $u: A \mapsto \mathbb{R}$ (the left and right extreme of $x$ respectively) such that $\forall x l(x)<u(x)$. Such a representation is equivalent to the one where to each element $x$ of $A$ is associated a function $g(x)$ and threshold function $t(x)$. We have $l(x)=g(x)$ and $u(x)=g(x)+t(x)$. In the rest of the paper we will only use the $(l(x), u(x))$ representation. Given the finite structure of set $A$, when we compare intervals we can restrict inequalities to their strict part without loss of any generality.

Further we consider a structure of two binary relations $P \subseteq A \times A$ and $I \subseteq A \times A$ (respectively named preference and indifference). From a data analysis point of view we can consider indifference as a similarity relation and the union of preference and its inverse as a dissimilarity relation. Hereafter, for sake of simplicity, we will only use the terms of preference and indifference such that: $P$ is asymmetric and irreflexive, $I$ is symmetric and reflexive, $P \cup I$ is complete and $P \cap I=\emptyset$.

Given any two binary relations $V, W$ on the set $A$ we denote $V . W(x, y)$ if and only if $\exists z: V(x, z) \wedge W(z, y)$. We denote $V \subseteq W$ for the formula $\forall x, y V(x, y) \Rightarrow W(x, y)$.

We are now able to give some basic definitions and theorems.

Definition 1. [2]. A $\langle P, I\rangle$ preference structure on a set $A$ is a PI interval order iff $\exists l, u: A \mapsto \mathbb{R}$ such that:

$\forall x: u(x)>l(x)$;

$\forall x, y: P(x, y) \Leftrightarrow l(x)>u(y)$

$\forall x, y: I(x, y) \Leftrightarrow l(x)<u(y)$ and $l(y)<u(x)$.

Definition 2. [2]. A $\langle P, I\rangle$ preference structure on a set $A$ is a PI semi order iff $\exists l$ : $A \mapsto \mathbb{R}$ and a positive constant $k$ such that:

$\forall x, y: P(x, y) \Leftrightarrow l(x)>l(y)+k ;$

$\forall x, y: I(x, y) \Leftrightarrow|l(x)-l(y)|<k$.

Such structures have been extensively studied in the literature (see for example [2]). We recall here below the two fundamental results which characterise interval orders and semi orders.

Theorem 1. [2]. A $\langle P, I\rangle$ preference structure on a set $A$ is a PI interval order iff P.I.P $\subset$ P.

Theorem 2. [2]. A $\langle P, I\rangle$ preference structure on a set $A$ is a $P I$ semi order iff P.I.P $\subset$ $P$ and I.P.P $\subset P$. 


\section{$3 \quad P Q I$ interval orders}

Recently [4] suggested that, while the conditions under which the relation $P$ holds could be considered fixed, the conditions under which the relation $I$ holds contain two different situations: One called "indifference" (where one interval is included to the other) and the other called "weak preference" or "hesitation between indifference and preference" (where the intersection of the two intervals is non empty, but one interval is "more to the right of the other"). More formally we consider preference structures composed of three preference relations: $P$ (which is asymmetric and irreflexive), $Q$ (which is asymmetric and irreflexive) and $I$ (which is symmetric and reflexive), $P \cup$ $Q \cup I$ being complete and mutual intersections being empty and we have the following results.

Definition 3. [4] A $\langle P, Q, I\rangle$ preference structure on a finite set $A$ is a $P Q I$ interval order, iff $\exists l, u: A \mapsto \mathbb{R}$ such that, $\forall x, y \in A, x \neq y$ :

$-u(x)>l(x)$;

$-P(x, y) \Leftrightarrow u(x)>l(x)>u(y)>l(y)$;

- $Q(x, y) \Leftrightarrow u(x)>u(y)>l(x)>l(y)$;

- $I(x, y) \Leftrightarrow u(x)>u(y)>l(y)>l(x)$ or $u(y)>u(x)>l(x)>l(y)$.

Theorem 3. [4] A $\langle P, Q, I\rangle$ preference structure on a finite set $A$ is a PQI interval order, iff there exists a partial order $I_{l}$ such that:

i) $I=I_{l} \cup I_{u} \cup I_{o}$ where $I_{o}=\{(x, x), x \in A\}$ and $I_{u}=I_{l}^{-1}$;

ii) $\left(P \cup Q \cup I_{l}\right) P \subset P$;

iii) $P\left(P \cup Q \cup I_{u}\right) \subset P$;

iv) $\left(P \cup Q \cup I_{l}\right) Q \subset P \cup Q \cup I_{l}$;

v) $Q\left(P \cup Q \cup I_{u}\right) \subset P \cup Q \cup I_{u}$.

Definition 4. [4] A PQI semi order is a PQI interval order such that $\exists k>0$ constant for which $\forall x: u(x)=l(x)+k$.

In other words, a $P Q I$ semi order is a $\langle P, Q, I\rangle$ preference structure for which there exists a real valued function $l: A \mapsto \mathbb{R}$ and a positive constant $k$ such that $\forall x, y$ :

$-P(x, y) \Leftrightarrow l(x)>l(y)+k$

$-Q(x, y) \Leftrightarrow l(y)+k>l(x)>l(y)$

$-I(x, y) \Leftrightarrow l(x)=l(y)$; (in fact $I$ reduces to $I_{o}$ ).

Theorem 4. [4] A $\langle P, Q, I\rangle$ preference structure is a $P Q I$ semi order iff:

i) $I$ is transitive

ii) $P P \cup P Q \cup Q P \subset P$;

iii) $Q Q \subset P \cup Q$;

\section{Valued Hesitation}

The existence of a zone of hesitation between strict preference and indifference and the introduction of valued relations in order to take in account such an hesitation has been first considered by Roy $([8,9])$ in the case of the so-called pseudo-orders and 
extensively studied in [10]. However, in this case they consider preference structures with two thresholds which is equivalent to a representation with intervals whose length is within an interval of values. The hesitation occurs between the extremities of this second interval.

In our case we consider preference structures with only one threshold. The hesitation is due to the interval structure of the information associated to each object. The above results however, although introduce the idea that comparing objects represented by intervals implies the existence of a zone of hesitation between preference and indifference, are unable to give a "measure" of such an hesitation.

Consider three objects whose cost is for the first $(x)$ in the interval $[10,18]$, for the second $(y)$ in the interval $[11,20]$ and for the third $(z)$ in the interval $[17,20]$. Using the previous approach we get $Q(x, y), Q(x, z)$ and $I(y, z)$. However, it is intuitively clear that the hesitation which occurs when objects $x$ and $y$ are compared is not the same with the hesitation which occurs when objects $x$ and $z$ are compared. Moreover, although objects $y$ and $z$ are considered indifferent it is again intuitively clear that they are indifferent to some extend and not identical.

The basic idea introduced is that the extend to which the two intervals have a non empty intersection could be a "measure" of the hesitation between preference and indifference. Such an idea dates back to Dubois and Prade ([11]), but applied to conventional preference structures where a distribution of possibility can be associated to alternatives under the form of a fuzzy number. In this approach we consider flat distributions of uncertainty in the sense that any value of the interval has the same possibility to represent the "real" value. From this point of view it is meaningful to compare lengths of intervals in order to have a "measure" of the uncertainty. The approach however, easy generalises in the case of specific uncertainty distributions.

First of all we will present a general framework for the fuzzification of preference structures having the three relations $P, Q$ and $I$ and then we will propose a model in order to calculate the evaluation of each relation.

We begin by introducing some basic notions and notations: We are going to use the symbols of $T$ and $S$ in order to represent T-norms and T-conorms respectively as continuous representations of conjunction and disjunction operators in the case of continuous valuations. $n$ will represent the negation operator (for a discussion about such operators in the frame of fuzzy sets theory see [12]).

T-norms and T-conorms are related by duality. Pairs of t-norms and t-conorms satisfy the generalisation of the De Morgan law as in the following:

Definition 5 (De Morgan Triplets ). Let $T$ be a t-norm, $S$ a t-conorm and $n$ a strict negation then $\langle T, S, n\rangle$ is a De Morgan triple iff $\forall x, y \in[0,1]$ :

$$
n(S(x, y))=T(n(x), n(y)) .
$$

Several De Morgan Triplets have been suggested in the literature. Zadeh and Lukasiewicz triplets (see [12]) are the most used ones in preference modelling. We denote them respectively by $\left(T_{\min }, S_{\min }, n\right)$ and $(L T, L S, n)$ (see Table 4$)$. The extension of the properties of binary relations in the valued case is straightforward and omitted for sake of space in this paper. The reader can see the relevant details in [13] and [14]. We only introduce in the following two notions that we need for the rest of the paper: 


\begin{tabular}{lcc}
\hline Names & $t$-norms & $t$-conorms \\
\hline Zadeh & $\min (x, y)$ & $\max (x, y)$ \\
Lukasiewicz & $\max (x+y-1,0)$ & $\min (x+y, 1)$ \\
\hline
\end{tabular}

Table 1. De Morgan triplets

Definition 6 (Zero divisor). [15] An element $x \in] 0,1[$ is called a zero divisor of a t-norm $T$ iff $\exists y \in] 0,1[T(x, y)=0$. A t-norm without zero divisors is called positive.

Definition 7 (Archimedean). [15] A continuous t-norm $T$ is Archimedean iff $\forall x \in$ ] $0,1[T(x, x)<x$.

It is easy to see that Lukasiewicz t-norms have a zero divisor and are Archimedean and that the minimum operator is positive.

We are ready now to give the characterisation of a fuzzy preference structure having $P, Q$ and $I$ that we call fuzzy $\langle P, Q, I\rangle$ preference structure. Our study is inspired from the work of De Baets et al. ([16]) concerning the case of partial preference structures fuzzification.

Definition 8 (Fuzzy $\langle P, Q, I\rangle$ preference structure). Consider a de Morgan triplet $M=(T, S, n)$ and three valued binary relations $P, Q$ and $I . A\langle P, Q, I\rangle$ structure on the set $A$ is a fuzzy preference structure w.r.t. $M$ iff:

1. $P$ and $Q$ are irreflexive and $T$-asymmetric,

2. $I$ is reflexive and symmetric,

3. $T(P, I)=0, T(P, Q)=0$ and $T(Q, I)=0$,

4. $S(P, S(Q, I))$ is $S$-complete.

We show by the help of the two next propositions that in order to have a real fuzzy preference structure defined in the whole unit interval $[0,1]$, continuous, Archimedean t-norms having zero divisors must be used.

Proposition 1. Consider a de Morgan triplet $M=(T, S, n)$ with a t-norm without zero divisors. If all the conditions of definition 8 are satisfied for a fuzzy $\langle P, Q, I\rangle$ structure with $M$, then the fuzzy relations $P, Q$ and I are crisp.

\section{Proof. see [17].}

Hence, it is better to have a zero divisor in order to have fuzzy relations. In this case we know another result concerning non-Archimedean t-norms.

Proposition 2. Consider a de Morgan triplet $M=(T, S, n)$ with a continuous nonArchimedean t-norm with zero divisors. If all the conditions of definition 8 are satisfied for a fuzzy $\langle P, Q, I\rangle$ structure with $M$, then $\exists x_{\varsigma} \in[0,1[$ such that $\forall x, y$,

$$
\begin{gathered}
p(x, y)<1 \Longrightarrow p(x, y)<x_{\varsigma}, \\
q(x, y)<1 \Longrightarrow q(x, y)<x_{\varsigma}, \\
i(x, y)<1 \Longrightarrow i(x, y)<x_{\varsigma} .
\end{gathered}
$$


Proof. see [17].

Hence, in order to use the whole unit interval $[0,1]$, continuous, Archimedean tnorms having zero divisors must be used. Such t-norms are called nilpotent and are $\phi$ transform of the Lukasiewicz t-norm. For that reason an alternative definition of fuzzy $\langle P, Q, I\rangle$ preference structure can be given:

Definition 9 (L-Fuzzy $\langle P, Q, I\rangle$ preference structure). Consider a Lukasiewicz triplet $L=(L T, L S, n)$ and three valued binary relations $P, Q$ and $I . A\langle P, Q, I\rangle$ structure on the set $A$ is a L-fuzzy preference structure w.r.t. $L$ if:

1. $P$ and $Q$ are irreflexive and LT-asymmetric,

2. $I$ is reflexive and symmetric,

3. $\operatorname{LT}(P, I)=0, L T(P, Q)=0, L T(Q, I)=0, L T\left(P, Q^{-1}\right)=0$, (exclusivity),

4. $L S(P, L S(Q, I))$ is $L S$-complete.

Although the definition 9 utilizes the whole unit interval, it presents some weaknesses. Three critics can be done:

1. The asymmetry condition with a t-norm having zero divisor allows the co-existence of strictly positive $p(x, y)$ and $p(y, x)$ for the same couple which can be contradictory to the semantics of preference relation or must be avoided in some situations.

2. The exclusivity condition with a t-norm having zero divisor do not permit us to forbid the co-existence of relations with contradictory semantics. For example, depending on the context of preference modelling, one may want to forbid the coexistence of $P$ and $Q^{-1}$, since in the majority of cases if there some credibility for the sentence " $x$ is strictly preferred to $y$ ", the sentence " $y$ is weakly preferred to $x$ " should not be credible at all.

3. Finally, within some context or for some decision maker it may be important to have a stronger completeness, in the sense that for every couple $x, y$, the characteristic relation or its converse should take the value 1 . Again, positive t-conorms do not satisfy such conditions.

For all these reasons, we propose the use of different triangular norms for each condition.

Definition 10 (Flexible Fuzzy $\langle P, Q, I\rangle$ preference structure). Consider De Morgan triplets $M_{i}=\left(T_{i}, S_{i}, n_{i}\right)$ with $i \in\{1, \ldots, 6\} . A\langle P, Q, I\rangle$ structure on the set $A$ is a flexible fuzzy preference structure w.r.t $M_{i}$ if:

1. $P$ and $Q$ are irreflexive, $P$ is $T_{1}$-asymmetric and $Q$ is $T_{2}$-asymmetric,

2. $I$ is reflexive and symmetric,

3. $T_{3}(P, I)=0, T_{4}(P, Q)=0, T_{5}(Q, I)=0$ and $T_{6}\left(P, Q^{-1}\right)=0$, (exclusivity),

4. $S_{1}\left(P, S_{1}(Q, I)\right)$ is $S_{1}$-complete. 
We define the characteristic relation $R$ as $R=S_{1}\left(P, S_{1}(Q, I)\right)$.

Before analysing all the possibilities concerning the use of different t-norms, we make some assumptions:

i. The asymmetry conditions use the min operator: $T_{1}=T_{2}=\min$,

ii. The exclusivity between $P$ and $Q^{-1}$ makes use of the min operator: $T_{6}=\min$,

iii. $\forall x, y$, if $r(x, y)=1$ and $r(y, x)=0$ then $p(x, y)=1, q(x, y)=0, i(x, y)=0$,

iv. $\forall x, y$, if $r(x, y)=1$ and $r(y, x)=1$ then $i(x, y)=1, p(x, y)=0, q(x, y)=0$.

Assumptions $i$ and $i i$ are related to the critics 1 and 2 and assumptions $i i i$ and $i v$ are natural conditions very frequently used in preference modelling and decision analysis. Unfortunately they are not sufficient to conclude our analysis. The determinations of $T_{3}, T_{4}, T_{5}$ and $S_{1}$ are less natural and need more detailed study.

We begin by the completeness condition. Considering the characteristic relation $R$ one can distinguish three different states: $r(x, y)=1, r(x, y)=0$ and $0<r(x, y)<1$. $R$ is $S_{1}$-complete means that $\forall x, y, S_{1}(R(x, y), R(y, x))=1$. Therefore, we analyse different permutations of the states of $R$ and $R^{-1}$ and we obtain nine cases where some of them are already defined by the previous assumptions (see Table 2).

\begin{tabular}{l||l|l|l|}
$R \backslash R^{-1}$ & 0 & ] $0,1[$ & 1 \\
\hline \hline 0 & $?$ & $?$ & $P^{-1}$ \\
\hline$] 0,1[$ & $?$ & $?$ & $?$ \\
\hline 1 & $P$ & $?$ & $I$ \\
\hline
\end{tabular}

Table 2. Values of $p, q$ and $i$ whit our hypotheses.

Now we are interested in unknown cases. For the completeness condition there are two possibilities. $S_{1}$ may be positive $\left(S_{1}^{+}\right)$or negative ${ }^{3}\left(S_{1}^{-}\right)$:

- If $S_{1}$ is positive, then

- if $r(x, y)=0$ then $r(y, x)=1$ because $R$ is $S$-complete: $S_{1}^{+}(r(x, y), r(y, x))=$ $S_{1}^{+}(0, r(y, x))=r(y, x)=1$,

- if $r(x, y)=1$ then $0 \leq r(y, x) \leq 1$ because in this case there is no condition on $r(y, x)$,

- if $0<r(x, y)<1$ then $r(y, x)=1$ because of the definition of positive t-norms.

These results allow us to complete some cases of the Table 2, especially the ones which do not satisfy the completeness condition and we obtain the first collum of Table 3.

- If $S_{1}$ is negative, then

- if $r(x, y)=0$ then $r(y, x)=1$ because $R$ is $S$-complete: $S_{1}^{-}(r(x, y), r(y, x))=$ $S_{1}^{-}(0, r(y, x))=r(y, x)=1$,

- if $r(x, y)=1$ then $0 \leq r(y, x) \leq 1$ because in this case there is no condition on $r(y, x)$,

\footnotetext{
${ }^{3}$ a De Morgan triplet with an involutive negation which is not positive
} 
- if $0<r(x, y)<1$ then $0<r(y, x) \leq 1$ because $S_{1}^{-}$is negative and has a zero divisor.

Like in the previous case, we complete the Table 2 thanks to these results and we obtain the second collum of Table 3 .

\begin{tabular}{l||l|l|l|}
$R \backslash R^{-1}$ & 0 & $0,1[$ & 1 \\
\hline \hline 0 & incomplete & incomplete & $P^{-1}$ \\
\hline$] 0,1[$ & incomplete & incomplete & $?$ \\
\hline 1 & $P$ & $?$ & $I$ \\
\hline
\end{tabular}

\begin{tabular}{l||l|l|l|}
$R \backslash R^{-1}$ & 0 & ] $0,1[$ & 1 \\
\hline \hline 0 & incomplete & incomplete & $P^{-1}$ \\
\hline$] 0,1[$ & incomplete & $?$ & $?$ \\
\hline 1 & $P$ & $?$ & $I$ \\
\hline
\end{tabular}

Table 3. Values of $p, q$ and $i$ when $r$ is $S^{+}$-complete and $S^{-}$-complete.

In order to complete our analysis, exclusivity conditions must be studied for the five cases expressed in Table 3 by "? ". Before beginning the analysis, let us mention that some symmetries and similarities can help us to decrease the number of cases to analyse: cases $(r(x, y)=1,0<r(y, x)<1)$ and $(r(y, x)=1,0<r(x, y)<1)$ are symmetric and provide similar results. Thus, analysing the cases $(r(x, y)=1,0<$ $r(y, x)<1)$ for positively and negatively completeness and $(0<r(x, y)<1,0<$ $r(y, x)<1)$ for positively completeness will be sufficient in order to finalise our analysis.

As in the completeness condition, for each t-norm of exclusivity conditions $\left(T_{3}, T_{4}\right.$ and $T_{5}$ ), there are two possibilities: $T_{i}(i=3,4,5)$ can be positive (and will be denoted by $T_{i}^{+}$) or negative (and will be denoted by $T_{i}^{-}$). All the permutation of these two possibilities for three t-norms (there are $2^{3}=8$ of them) are analysed and the results are presented in Table 4 (the detailed analysis of these cases are not presented in this paper for the sake of space, an interested reader can see in [17]). Remark that in each case of Table 4 we present only relations which can be valued. For example having an empty case, like the first case of the first line, means that in this case we can only have crisp relations. When there are some relations in a case, like the second column of the first line, it means that these relations can be valued while the remaining ones are crisp. In the case of the second column of the first line, the indifference can be valued while $P$ and $Q$ are crisp.

Briefly, we remark in Table 4 that lines 1 and 3 correspond to crisp $\langle P, Q, I\rangle$ preference structures or $\langle P, Q, I\rangle$ preference structures having only the indifference relation as a valued one. Cases 6 and 8 do not have a natural interpretation. Lines 2, 7, 4 and 5 provide fuzzy $\langle P, Q, I\rangle$ preference structures with one crisp and two valued relations. In lines 2 and 7, there are some relative positions having $Q=1$ and some others $0<I \leq 1$ and $0<P \leq 1$ while lines 4 and 5 provide some relative positions having $P=1$ and some others $0<I \leq 1$ and $0<Q \leq 1$. The interpretations of the two first cases are not so natural (how to interpret the fact of having a valued "strict preference" while the weak preference is always crisp?). With lines 4 and 7 , the strict preference appears as an upper bound of the weak preference relation where the preference becomes sure. For all these reasons lines 4 and 5 appear as the most favorable ones, between the eight ones, in order to fuzzify $\langle P, Q, I\rangle$ preference structures. Such structures are strongly related 


\begin{tabular}{|c|c|c|}
\hline Exclusivity & $\begin{array}{c}\text { Positively or negatively complete } \\
\text { and } \\
r(x, y)=1,0<r(y, x)<1\end{array}$ & $\begin{array}{c}\text { Positively complete } \\
\text { and } \\
0<r(x, y)<1,0<r(y, x)<1\end{array}$ \\
\hline $\begin{array}{c}1) \\
T_{3}^{+}(P, I)=0 \\
T_{4}^{+}(P, Q)=0 \\
T_{5}^{+}(Q, I)=0\end{array}$ & & $I$ \\
\hline $\begin{array}{c}2) \\
T_{3}^{-}(P, I)=0 \\
T_{4}^{+}(P, Q)=0 \\
T_{5}^{+}(Q, I)=0\end{array}$ & $P, I$ & $P, I, P^{-1}$ \\
\hline $\begin{array}{c}3 \text { 3) } \\
T_{3}^{+}(P, I)=0 \\
T_{4}^{-}(P, Q)=0 \\
T_{5}^{+}(Q, I)=0 \\
\end{array}$ & & $I$ \\
\hline $\begin{array}{c}4) \\
T_{3}^{+}(P, I)=0 \\
T_{4}^{+}(P, Q)=0 \\
T_{5}^{-}(Q, I)=0\end{array}$ & $Q, I$ & $Q, I, Q^{-1}$ \\
\hline $\begin{array}{c}5) \\
T_{3}^{+}(P, I)=0 \\
T_{4}^{-}(P, Q)=0 \\
T_{5}^{-}(Q, I)=0\end{array}$ & $Q, I$ & $Q, I, Q^{-1}$ \\
\hline $\begin{array}{c}6) \\
T_{3}^{-}(P, I)=0 \\
T_{4}^{+}(P, Q)=0 \\
T_{5}^{-}(Q, I)=0 \\
\end{array}$ & $\begin{array}{l}P, I \\
Q, I\end{array}$ & $\begin{array}{l}P, I, P^{-1} \\
Q, I, Q^{-1}\end{array}$ \\
\hline $\begin{array}{c}7) \\
T_{3}^{-}(P, I)=0 \\
T_{4}^{-}(P, Q)=0 \\
T_{5}^{+}(Q, I)=0\end{array}$ & $P, I$ & $P, I, P^{-1}$ \\
\hline $\begin{array}{c}8) \\
T_{3}^{-}(P, I)=0 \\
T_{4}^{-}(P, Q)=0 \\
T_{5}^{-}(Q, I)=0\end{array}$ & $P, Q, I$ & $P, Q, I, Q^{-1}, P^{-1}$ \\
\hline
\end{tabular}

Table 4. Relations which can be valued when different $T_{3}, T_{4}, T_{5}$ and $S_{1}$ are used.

to fuzzy $\langle P, I\rangle$ preference structures but may have additional utility for the construction of models where the strict preference needs to be marked strongly.

As an example, we propose in the following a model used for the comparison of intervals where the situation of two disjoint intervals must be presented by a crisp strict preference relation, while other situations may have valued presentation.

Let $x$ and $y$ be two elements of a finite set A, having an interval representation such that $x:[l(x), u(x)]$ and $y:[l(y), u(y)]$ with $\forall x, l(x)<u(x)$. We define first of all the characteristic relation of our model: 
Definition 11. The credibility of the assertion " $x$ is at least as good as $y(r(x, y))$ " is such that

$$
\forall x, y \in A, r(x, y)=\max \left(0, \min \left(1, \max \left(\frac{u(x)-l(y)}{u(y)-l(y)}, \frac{u(x)-l(y)}{u(x)-l(x)}\right)\right)\right) .
$$

Different values of the relation $R$ for different interval comparison case are presented in Table 5.

The characteristic relation covers the strict preference, the indifference and the hesitation among the two previous relations. The second step is to define all these valued relations in terms of $R$. For this purpose we make use of the symmetric and the asymmetric part of $R$ :

$R^{a}(x, y)=\min \left[1, \max \left(0, \min \left(\frac{u(x)-u(y)}{u(x)-l(x)}, \frac{l(x)-l(y)}{u(y)-l(y)}\right)\right)\right]$,
$R^{s}(x, y)=\frac{\max [0,(\min (u(x)-l(y), u(x)-l(x), u(y)-l(y), u(y)-l(x)))]}{\min (u(x)-l(x), u(y)-l(y))}$

As in the crisp case, $R$ is the union of $P, Q$ and $I$ where $P, Q$ are asymmetric and $I$ is symmetric. For that reason, we propose to define $R^{a}$ and $R^{s}$ such as:

$$
R^{a}=S(P, Q) \text { and } R^{s}=I .
$$

The indifference can be directly obtained from equation 2. For $P$ and $Q$ we have to separate the relation $R^{a}$. For this purpose we add a new hypothesis which says that the strict preference $P$ exists only in the case of two disjoint intervals:

$$
\forall x, y \in A, p(x, y) \Longleftrightarrow l(x) \geq u(y) .
$$

As a result we define our three relations as in the following:

$$
\begin{gathered}
p(x, y)= \begin{cases}R_{I}^{a}(x, y) & \text { if } l(x) \geq u(y), \\
0 & \text { ifnot; }\end{cases} \\
q(x, y)= \begin{cases}R_{I}^{a}(x, y) & \text { if } l(x)<u(y), \\
0 & \text { ifnot; }\end{cases} \\
i(x, y)=R_{I}^{s}(x, y) .
\end{gathered}
$$

Table 5 illustrates all the values of the three relations for each interval comparison case.

Such a model has nice properties:

Proposition 3. Suppose that a binary relation $P$ and valued relations $Q$ and $I$ are defined as in equations 3-5, then 


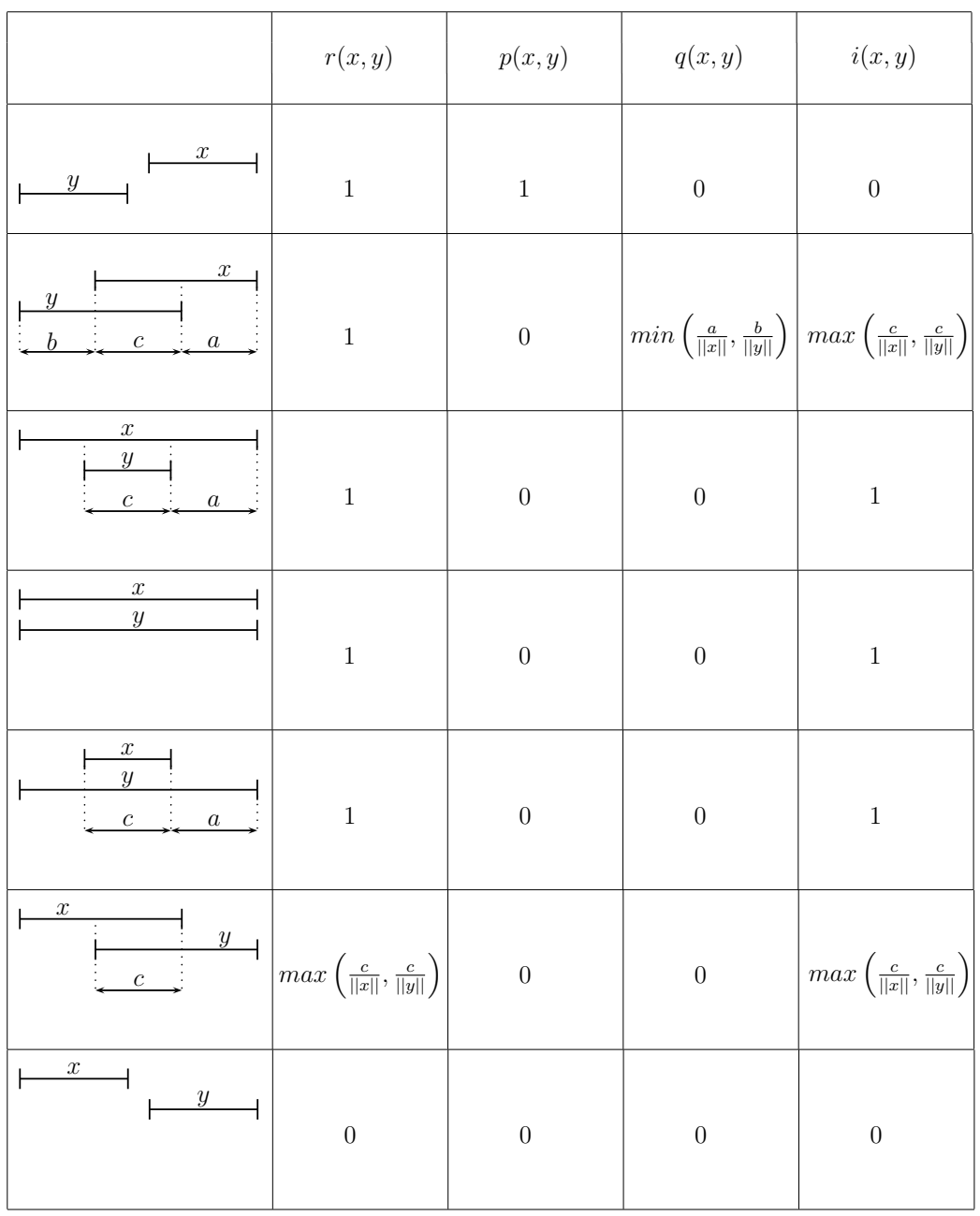

Table 5. Relations of the interval comparison with valued hesitation

i. $P$ and $Q$ are irreflexive and min-asymmetric,

ii. $I$ is reflexive and symmetric,

iii. $T_{\min }(P, I)=0, T_{\min }(P, Q)=0, L T(Q, I)=0, T_{\min }\left(P, Q^{-1}\right)=0$, iv. $L S(P, L S(Q, I))$ is $L S$-complete.

Proof. see [17].

The relations between the characteristic relation $R$ and the three preference relations are presented in the following proposition.

Proposition 4. Suppose that the three valued relations $P, Q$ and I are defined as in equations 3-5, then: 


$$
\begin{aligned}
& \text { i. } L S(P, Q)=R^{d} \\
& \text { ii. } I=\min \left(R, R^{-1}\right) \\
& \text { or explicitly, } \forall(x, y) \in A \times A \\
& \quad \text { i. } p(x, y)+q(x, y)=1-r(y, x) \\
& \quad \text { ii. } i(x, y)=\min \{r(x, y), r(y, x)\}
\end{aligned}
$$

Proof. see [17].

\section{Discussion}

What do we get with such results? What can we do with such valued relation? We consider two cases.

The first, obvious, case concerns the domain of preference modelling. Having a functional representation of the type described in the above section enables to give an explicit representation of the uncertainty and hesitation which appears when we compare intervals and to overcome the difficulty associated to the use of crisp thresholds. In fact if a discrimination problem exists this will concern any type of comparison. Therefore even if we fix a discrimination threshold there always exists an interval around the threshold for which a discrimination problem has to be considered (and that recursively for any new threshold introduced). The valued representation solves this problem. In this particular case the solution does not require the introduction of two thresholds, but gives a valued version for preference and indifference in all cases intervals are compared.

The second case concerns more generally the problem of comparing objects not necessarily for preference modelling reasons. As already introduced we can always consider the concept of indifference equivalent to the one of similarity, the concept of preference becoming a directed dissimilarity. Establishing the similarity among objects is a crucial problem for several research fields such as statistics, data analysis (in archeology, geology, medical diagnosis etc.), information theory, classification, case based reasoning, machine learning etc. A specific area of interest in the use of similarity relations is in rough sets theory ([18]).

In rough sets we consider objects described under a set of attributes and we establish a relation of indiscernibility (which is a crisp equivalence relation) in order to take in account our limited descriptive capability. In other terms real objects might be different, but due to our limited descriptive capability (represented by the set of attributes) we might be obliged to consider them as identical (indiscernible). Indiscernibility classes are then used in order to induce classification rules. However, equivalence relations can be very restrictive for several real cases where the more general concept of similarity is more suitable (see $[19,20]$ ). The use of a valued similarity has been considered in [21-24] for several different cases. Thanks to such a relation it is possible to induce classification rules to which a credibility degree is associated. By this way it is possible to enhance the classification capability of a data set although a confidence degree inferior to 1 has to be accepted. The approach described in this paper enables to give a theoretical foundation for the case where objects have to compared on attributes with continuous scales and where either a discrimination threshold has to be considered or the objects are represented by intervals. 


\section{Conclusion}

In this paper we present some results concerning the extension of $P Q I$ interval orders under continuous valuation. Particularly we propose a general frame work for the characterization of fuzzy preference structures with $P, Q$ and $I$ and give the functional representation for these three relations such that the portion of interval which is common is considered as a "measure" of the hesitation associated to the interval comparison. Such functions fulfill a number of nice properties in the sense that they correspond to a fuzzy preference structure as defined in [14].

The use of such valued preference relations not only enhance the toolkit of preference modelling, but enables a more flexible representation in all cases where a similarity among objects is under question. The particular case of rough sets theory is discussed in the paper. Several research directions remain open such as:

- the problem of aggregating such valued relations in order to obtain a comprehensive relation (crisp or valued) when several attributes or criteria are considered;

- a further analysis of the formal properties fulfilled by such valued relations;

- the analysis of such preference structures under the positive/negative reasons framework as introduced in [25] and discussed in [26].

\section{References}

1. Luce, R.D..: Semiorders and a theory of utility discrimination. Econometrica 24 (1956) 178-191

2. Fishburn, P.C..: Interval Orders and Interval Graphs. J. Wiley, New York (1985)

3. Pirlot, M., Vincke, Ph..: Semi Orders. Kluwer Academic, Dordrecht (1997)

4. Tsoukiàs, A., Vincke, $\mathrm{Ph} .$. : A characterization of pqi interval orders. Discrete Applied Mathematics 127(2) (2003) 387-397

5. Ngo The, A., Tsoukiàs, A., Vincke, Ph..: A polynomial time algorithm to detect PQI interval orders. International Transactions in Operational Research 7 (2000) 609-623

6. Ngo The, A., Tsoukiàs, A.: Numerical representation of pqi interval orders. Discrete Applied Mathematics 147 (2005) 125-146

7. Oztürk, M., Tsoukiàs, A.: Positive and negative reasons in interval comparisons: Valued pqi interval orders. In: Proceedings of IPMU 2004. (2004) 983-989

8. Roy, B., Vincke, Ph..: Relational systems of preference with one or more pseudo-criteria: Some new concepts and results. Management Science 30 (1984) 1323-1335

9. Roy, B., Vincke, Ph.: Pseudo-orders: definition, properties and numerical representation. Mathematical Social Sciences 14 (1987) 263-274

10. Perny, P., Roy, B.: The use of fuzzy outranking relations in preference modelling. Fuzzy Sets and Systems 49 (1992) 33-53

11. Dubois, D., Prade, H.: Decision making under fuzziness. In: Advances in fuzzy set theory and applications, North Holland, Amsterdam (1979) 279-302

12. Dubois, D., Prade, H.: Fuzzy sets and systems - Theory and applications. Academic press, New York (1980)

13. Fodor, J., Roubens, M.: Fuzzy preference modelling and multicriteria decision support. Kluwer Academic Publishers (1994)

14. Perny, P., Roubens, M.: Fuzzy preference modelling. In Słowiński, R., ed.: Fuzzy sets in decision analysis, operations research and statistics. Kluwer Academic, Dordrecht (1998) $3-30$ 
15. Ovchinnikov, S.N.: Modelling valued preference relation. In Kacprzyk, J., Fedrizzi, M., eds.: Multiperson decion making usingfuzzy sets and possibility theory. Kluwer, Dordrecht (1990) 64-70

16. Van De Walle, B., De Baets, B., Kerre, E.: Charaterizable fuzzy preference structures. Annals of Operations Research 80 (1998) 105-136

17. Ozturk, M.: Structures mathématiques et logiques pour la comparaison des intervalles. Thèse de doctorat, Université Paris-Dauphine (2005)

18. Pawlak, Z.: Rough Sets. Theoretical Aspects of Reasoning about Data. Kluwer, Dordrecht (1991)

19. Slowinski, R., Vanderpooten, D.: Similarity relation as a basis for rough approximations. In P., W., ed.: Advances in Machine Intelligence \& Soft-computing, Bookwrights, Raleigh (1997) 17-33

20. Slowinski, R., Vanderpooten, D.: A generalized definition of rough approximations based on similarity. IEEE Transactions on Data and Knowledge Engineering 12 (2000) 331-336

21. Greco, S., Matarazzo, B., Slowinski, R.: Handling missing values in rough set analysis of multi-attribute and multi-criteria decision problems. In in N. Zhong, Skowron, A., Ohsuga, S., eds.: New Directions in Rough Sets, Data Mining, and Granular-Soft Computing, LNAI 1711, Springer-Verlag, Berlin (1999) 146-157

22. Stefanowski, J., Tsoukiàs, A.: On the extension of rough sets under incomplete information. In Zhong, N., Skowron, A., Ohsuga, S., eds.: New Directions in Rough Sets, Data Mining and Granular-Soft Computing. Springer Verlag, LNAI 1711, Berlin (1999) 73-81

23. Stefanowski, J., Tsoukiàs, A.: Valued tolerance and decision rules. In Ziarko, W., Yao, Y., eds.: Rough Sets and Current Trends in Computing. Springer Verlag, LNAI 2005, Berlin (2001) 212-219

24. Stefanowski, J., Tsoukiàs, A.: Incomplete information tables and rough classification. Computational Intelligence 17 (2001) 454-466

25. Perny, P., Tsoukiàs, A.: On the continuous extension of a four valued logic for preference modelling. In: Proceedings of the IPMU 1998 conference, Paris. (1998) 302-309

26. Tsoukiàs, A., Perny, P., Vincke, Ph..: From concordance/discordance to the modelling of positive and negative reasons in decision aiding. In Bouyssou, D., Jacquet-Lagrèze, E., Perny, P., Słowiński, R., Vanderpooten, D., Vincke, Ph., eds.: Aiding Decisions with Multiple Criteria: Essays in Honour of Bernard Roy. Kluwer Academic, Dordrecht (2002) 147-174 